\title{
Evidências de validade das Matrizes Progressivas Avançadas de Raven em universitários
}

\author{
Milena Oliveira Rosseti - Casa do Psicólogo, Itatiba, Brasil \\ Ivan Sant' Ana Rabelo - Casa do Psicólogo, Itatiba, Brasil \\ Irene F. Almeida de Sá Leme - Casa do Psicólogo, Itatiba, Brasil \\ Silvia Verônica Pacanaro - Casa do Psicólogo, Itatiba, Brasil \\ Ingo Bernd Güntert - Casa do Psicólogo, Itatiba, Brasil
}

\begin{abstract}
Resumo
O objetivo do estudo foi encontrar evidências de validade das Matrizes Progressivas Avançadas de Raven em universitários. Participaram 369 universitários de duas universidades privadas do estado de São Paulo, sendo 104 $(28,2 \%)$ do sexo masculino e $265(71,8 \%)$ do feminino. Em relação aos cursos, 167 (45,3\%) são de Psicologia, 111 (30,1\%) de Administração de Empresas, 54 (14,6\%) de Gestão de Recursos Humanos e 37 (10\%) de Pedagogia. Os resultados demonstraram que houve diferença significativa com relação ao gênero, com um melhor desempenho do sexo masculino $(\mathrm{t}=4,21$ e $p<0,01)$. A análise de variância (ANOVA) mostrou que ocorreu diferença significativa entre as médias obtidas em cada curso $(F=13,8$ e $p<0,00)$, e os cursos que se diferenciaram de acordo com o teste ad-hoc de Tukey foram os de Administração e Psicologia. Os resultados demonstraram que o instrumento utilizado mostrou-se preciso para avaliar aspectos da inteligência geral em universitários.

Palavras-chave: inteligência; avaliação psicológica; ensino superior.
\end{abstract}

\section{Validity Evidence of Raven's Advanced Progressive Matrices in university students}

\begin{abstract}
The aim of the study was to find validity evidence of Raven's Advanced Progressive Matrices in university students. The sample consisted of 369 university students from two private universities in the state of Sao Paulo, being a 104 $(28,2 \%)$ of the masculine and $265(71,8 \%)$ of the feminine. Regarding the courses, $167(45,3 \%)$ is of Psychology, $111(30,1 \%)$ is of Business Administration, 54 (14,6\%) is of Management of Human Resources and $37(10 \%)$ is of Pedagogy. The results showed statistically significant difference of sex, with males having better performance in the instrument $(\mathrm{t}=4.21 ; p<0.01)$. The analysis of variance (ANOVA) showed significant difference between averages of the courses $(\mathrm{F}=13,8 ; p<0,00)$, and the courses that are differentiated in accordance with the test ad-hoc of Tukey were Administration and Psychology that obtained scores bigger than the others courses. The results demonstrated what the used instrument showed precisely to value aspects of the general intelligence at university students.

Keywords: intelligence; psychological assessment; higher education.
\end{abstract}

\section{Introdução}

A avaliação psicológica nos últimos anos vem se difundindo e trazendo muitas contribuições em diversas áreas do conhecimento da psicologia. Podese defini-la como um processo de coleta de dados e interpretações, por meio de informações obtidas em questionários, métodos, instrumentos psicológicos, entrevistas, entre outros (Noronha \& Alchieri, 2002; Pasquali, 2001; Primi, Flores-Mendoza \& Castilho, 1998; Wechsler, 1999). Em referência a instrumentos psicológicos, estes se caracterizam como uma forma objetiva para realizar a mensuração de determinadas características do comportamento humano, o que exige um processo cuidadoso de elaboração e verificação da maneira como essa atividade é conduzida (Alves, 2006; Muñiz, 1996). Os testes psicométricos, especificamente, fazem uso obrigatório da estatística e supõem uma medida dos traços psicológicos de uma pessoa, como, por exemplo, testes que medem a inteligência.

Um dos instrumentos mais utilizados para essa avaliação é o teste Matrizes Progressivas Coloridas de Raven (CPM), que foi construído para avaliar, em maior detalhe, os aspectos intelectuais de crianças de 5 a 11 anos. Além desta, existem também mais duas escalas do teste, que são Matrizes Progressivas de Raven Escala Geral - Standard Progressive Matrices (SPM) e as Avançadas - Advanced Progressive Matrices (APM) (Pasquali, 2002).

O presente estudo utilizou as Matrizes Progressivas Avançadas de Raven (APM), uma vez que estas apresentam poucos estudos de evidência de validade e precisão para a população brasileira, conforme destacou o Conselho Federal de Psicologia (2007).

O APM foi utilizado inicialmente em 1941, entrando em circulação restrita em 1947, sendo

\footnotetext{
${ }^{1}$ Endereço para correspondência:

Rua Simão Álvares, 1020, Vila Madalena - 05417-020 - São Paulo-SP, Brasil

E-mail:milena@casadopsicologo.com.br
} 
aplicado como medida de avaliação da inteligência não-verbal para pessoas com capacidade intelectual superior à média, destinado para avaliação de adolescentes (a partir de 12 anos) e adultos, como observação de progresso educacional, profissional, entre outros (Angelini, Alves, Custódio, Duarte \& Duarte, 1999). A escala foi elaborada por níveis de dificuldade dos itens em cada série. Na Série I são apresentados 12 problemas, usados para o treinamento, enquanto para a Série II, apresenta uma sequência de 36 problemas, em ordem de dificuldade crescente. Segundo Paín (1992), as Matrizes de Raven se apresentam como distintas de outros instrumentos de avaliação do construto inteligência. Enquanto alguns testes se utilizam de provas e jogos buscando abranger uma série de comportamentos muito diferentes a partir de estimulações, situações e instruções variadas, como nos testes Stanford-Binet IQ test, Escala de Inteligência Wechsler para Crianças (WISC) e Escala de Maturidade Mental Colúmbia (CMMS), o APM apresenta ao sujeito uma tarefa única e um material organizado sob a forma de figuras, chamado pelo autor de matrizes.

Nessa escala, destaca-se a presença de um fator geral (fator $g$ ) único e comum para o instrumento. Esse fator foi proposto por Spearman e definido como um nível intelectual comum a toda atividade mental, sendo uma capacidade básica do indivíduo em estabelecer relações abstratamente e que possibilita a pessoa lidar com aspectos característicos de cada situação problema (Primi \& Almeida, 2000). Spearman (1923, citado por Angelini \& colaboradores, 1999) identificou dois componentes do fator $g$, a saber, capacidade edutiva e reprodutiva. A capacidade edutiva envolve extrair significado de uma situação confusa, desenvolver novas compreensões e estabelecer construtos que facilitem lidar com problemas complexos, enquanto a capacidade reprodutiva inclui o domínio, a lembrança e a reprodução de materiais que constituem uma base cultural de conhecimentos explícitos.

As capacidades edutiva e reprodutiva foram observadas em estudo exploratório das propriedades psicométricas de uma versão experimental informatizada do APM. O estudo foi realizado por Primi, Flores-Mendonza e Castilho (1998), com a qual participação de 360 universitários, que responderam ao instrumento de forma coletiva, sem limite de tempo. Observou-se o coeficiente de precisão do instrumento para a série II, por meio da fórmula de Kuder-Richardson de 0,83 . No que se refere às notas escolares, a correlação com os escores da série I foi de $0,34(\mathrm{n}=86 ; p<0,01)$ e com os da série II de 0,41 $(\mathrm{n}=108 ; p<0,01)$. Os resultados permitiram concluir que a versão informatizada manteve propriedades psicométricas similares às da versão tradicional, mostrando a necessidade de aumentar estudos dessa natureza.

No que se refere aos estudos de evidência baseados nas relações com outras variáveis, Cunha (1974), utilizando a variável idade, afirma que a curva do crescimento intelectual do homem tem aceleração positiva na infância e na pré-adolescência, decrescendo sensivelmente na adolescência e tornando-se nula na idade adulta. Da idade madura para a velhice a curva apresentaria um declínio significativo. $\mathrm{Na}$ pesquisa de padronização do teste Wechsler Adult Intelligence Scale (WAIS) foi observado que o resultado máximo ocorria entre 20 e 30 anos, com um leve declínio até os 60 anos, acentuando-se a partir dessa idade (Anastasi, 1977). Entretanto, Matarazzo (1976) ressalta a função da escolarização nesse processo, considerando que os indivíduos mais velhos viveram em um meio educacional com experiências diferentes dos mais jovens, que em média tinham anos de escolaridade menores. Segundo o autor, esses dados levam à impressão de um declínio com a idade, que é apenas aparente, visto que as pesquisas longitudinais demonstraram que a inteligência do adulto continua a aumentar discretamente até os 50 anos e, somente então, começa a mostrar um pequeno declínio. Tomando por base esses resultados o autor lança a problemática da necessidade de atualizações constantes de normas dos testes, visando corrigir possíveis distorções.

Da mesma forma, Alves, Colosio e Ruivo (1992), utilizando o teste de inteligência não-verbal R-1, realizaram um levantamento em uma amostra de candidatos a emprego em uma empresa e verificaram a relação da escolaridade com a idade dos participantes. Foi observado que houve maior frequência de pessoas com escolaridade até o ensino básico (primário) entre 30 e 45 anos, com ensino fundamental entre 20 e 35 anos e com ensino médio entre 20 e 30 . Esses dados demonstram que os sujeitos com idade mais avançada possuem um grau de escolaridade menor e um declínio da pontuação do instrumento utilizado com relação à idade. Nesse sentido, Alves (1998) também verificou um declínio no número de pontos em teste de inteligência com o aumento da idade.

No que se refere à análise entre a variável gênero e inteligência em pessoas de escolaridade superior, Colom e Garciápz (2002) realizaram uma pesquisa com 4.072 alunos, sendo 1.772 mulheres e 2.300 homens. Foram utilizadas três medidas de inteligência: Catell's Culture-Fair Intelligence test, Inductive Reasoning Tests taken from the Primary Mental Abilities 
Battery (PMA-R) e Advanced Progressive Matrices (APM). Os resultados indicaram que não houve diferença significativa entre gênero no Catell's Culture-Fair Intelligence test. $\mathrm{O}$ sexo feminino obteve um desempenho mais elevado no $P M A-R$ ( $t=5.62$, $p<0,01)$ e o sexo masculino teve um melhor desempenho no $A P M(t=3.57, p<0,01)$.

De acordo com Weiss, Kemmler, Deisenhammer, Fleischhacker e Delazer (2003), avaliaram a magnitude das diferenças de gênero em uma bateria neuropsicológica, com foco nas habilidades verbais e espaciais visuais. Participaram 97 estudantes de ensino médio, sendo 51 do sexo feminino e 46 do masculino. Foram encontradas diferenças significativas entre gênero em três de quatro medidas verbais e em uma das três tarefas espaciais visuais. Os resultados mostraram nos testes verbais que as mulheres apresentaram um nível mais elevado do que os homens, enquanto estes tendem a superar as mulheres em tarefas espaciais visuais. Ressalta-se, entretanto que nesta pesquisa estas diferenças foram consideradas pequenas.

$\mathrm{Na}$ pesquisa realizada por Alves (2007) para atualizações no teste de inteligência D.70 verificou-se em uma amostra de 2.108 estudantes, sendo $1.966 \mathrm{em}$ universitários e 147 estudantes do ensino médio com idade entre 15 e 69 anos, que os participantes do sexo masculino do ensino superior obtiveram médias mais elevadas $(M=28,09)$ do que os do sexo feminino $(\mathrm{M}=25,78), \quad$ com relevância estatisticamente significativa $(p<0,01)$.

Sobre a influência das diferenças individuais no desempenho intelectual, Candeias e Almeida (2000) afirmam que as diferenças individuais no desempenho intelectual estão ligadas a uma maior relação da própria inteligência com o meio cultural, a aprendizagem e os contextos de vida de cada pessoa. Os resultados mais recentes na investigação no domínio da cognição sugerem ser necessário complementar os testes de QI e de fator $g$ com formas diferentes de avaliação da inteligência e das funções cognitivas. Os autores salientam que o desempenho cognitivo dos indivíduos reflete, simultaneamente, o tipo de processo (raciocínio, memória, motivação, criatividade, entre outros) e conteúdos mentais (verbal, espacial, numérico, entre outros) das tarefas a serem realizadas, mostrando que os níveis de habilidade e de desempenho se diferenciam diante das exigências de mudanças culturais, educacionais, laborais e do desempenho em geral, o que justifica um novo olhar para a inteligência.

Sendo assim, com o intuito de contribuir com estudos sobre aspectos da inteligência, o presente artigo tem como objetivo corroborar os estudos realizados sobre o tema, investigando evidências de validade das Matrizes Progressivas Avançadas de Raven, além de explorar possíveis diferenças, em universitários, relativas ao gênero, faixa etária e cursos entre as pontuações obtidas no teste.

\section{Método}

\section{Participantes}

A aplicação do instrumento para coleta dos dados foi realizada com 369 estudantes de ensino superior de duas universidades privadas no estado de São Paulo, sendo 297 (80,5\%) de uma universidade na cidade de São Paulo e $72(19,5 \%)$ de uma universidade de São José dos Campos selecionados em amostragem por conveniência. Dos participantes investigados $104(28,2 \%)$ eram do sexo masculino e $265(71,8 \%)$ do sexo feminino. As idades variaram entre 17 e 63 anos, com média de 28,1 anos e desvio padrão (DP) de 9,4.

Em relação aos participantes dos cursos, verificou-se que $167(45,3 \%)$ são alunos de Psicologia, 111 (30,1\%) de Administração de Empresas, 54 (14,6\%) de Gestão de Recursos Humanos e 37 (10\%) de Pedagogia.

\section{Instrumentos}

Matrizes Progressivas Avançadas de Raven (Raven, Raven \& Court, 1998).

O Teste das Matrizes Progressivas Avançadas de Raven (APM), padronizado e publicado em 1941, foi desenvolvido por Raven para abranger pessoas com capacidade intelectual superior à média, sendo mais usada para pessoas com escolaridade universitária. Destina-se à avaliação de adolescentes (a partir de 12 anos) e adultos.

A aplicação pode ser individual ou coletiva e o tempo máximo de realização é de 60 minutos. A aplicação do APM é realizada por meio de dois cadernos, denominados série I e II. A série I é composta por 12 itens e a série II por 36 itens. O nível de dificuldade aumenta a cada item em ambas as séries, e a série II possui um nível de dificuldade superior à serie I, que tem a função de servir como exemplo para a resolução da série seguinte. Em cada item é apresentado um quadro com uma figura na qual falta um pedaço. Abaixo do quadro são apresentadas oito opções com possíveis complementos para esse pedaço. O participante deve assinalar o pedaço que melhor completar a figura do quadro, anotando a opção na folha de resposta.

A correção do teste é feita atribuindo-se um ponto à resposta correta e zero à resposta 
considerada errada, variando a pontuação de 0 a 36 . Em seguida, soma-se a pontuação obtida e transformam-se os dados em percentil, observando as tabelas de padronização, para a localização do QI correspondente.

\section{Procedimentos}

Os aplicadores foram previamente treinados para as aplicações do instrumento, sendo utilizada somente a série II e seguido o procedimento padrão de aplicação. As aplicações ocorreram em dias agendados previamente com as universidades, conforme o cronograma de atividades de cada curso. Foram entregues para os alunos um Termo de Consentimento Livre e Esclarecido para que autorizassem a participação na pesquisa. Após a assinatura, os alunos dos cursos correspondentes preencheram o teste de forma coletiva. As aplicações duraram de aproximadamente 40 minutos.

\section{Análise dos dados}

As informações coletadas foram analisadas pelo Programa Statistical Package for the Social Sciences (SPSS), obtendo dados de precisão por meio do alfa de Cronbach, de medidas descritivas e de distribuição de frequência. Realizou-se por meio da correlação de Pearson um estudo com a variável idade e a pontuação (acertos) no teste. Para verificar possíveis diferenças de médias de pontos entre os grupos de faixas etárias realizou-se uma análise de variância (ANOVA). O nível de significância estabelecido foi de 5\%. Com relação ao gênero, realizou-se uma comparação entre as médias de pontos dos dois grupos por meio do Test $\mathrm{t}$ (Independent Sample). Para o estudo de evidências de validade baseados nas relações com outras variáveis, realizou-se uma análise de variância (ANOVA) entre as médias dos cursos frequentados pelos universitários com posterior aplicação da Prova de Tukey, uma vez que foi encontrada diferença estatisticamente significante.

\section{Resultados}

Considerando os objetivos do presente estudo, as informações a seguir referem-se aos resultados obtidos a partir da pontuação total da APM. Inicialmente foi verificada a precisão do instrumento, e em seguida foram utilizadas variações de médias de pontos em relação à faixa etária, gênero e curso. No que se refere à precisão do APM, verificou-se um coeficiente alfa de Cronbach de 0,87. Como o índice apresentou-se satisfatório, isto é, superior a 0,80 , pode-se inferir que o teste possui um bom nível de precisão, sendo considerado um instrumento confiável para a medida do respectivo construto nesse grupo analisado (Cronbach, 1996). Na Figura 1 é apresentada a distribuição da frequência de pontos no teste APM.

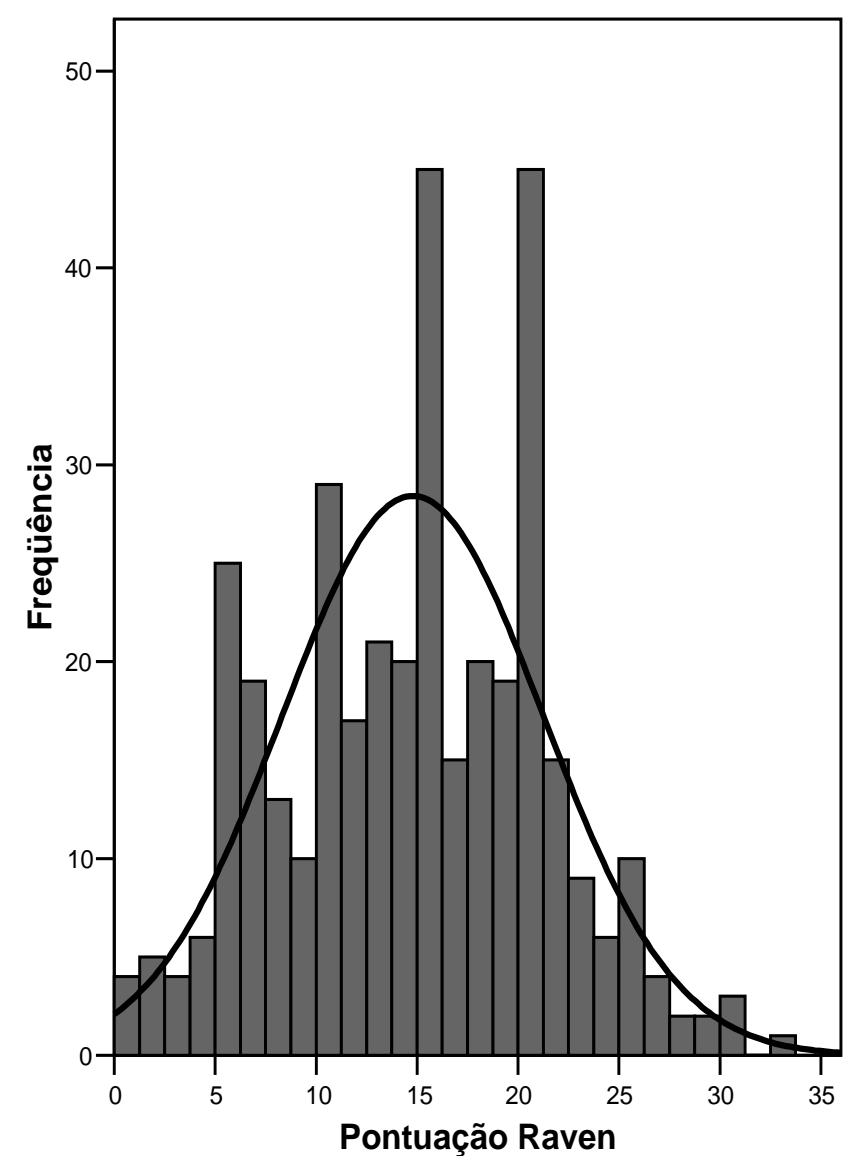

Figura 1 - Frequência de pontos no teste

Observou-se que a maior parte dos participantes (56,1\%) apresentou entre 10 e 20 acertos, sendo os demais distribuídos em 18,2\% com pontuação entre 5 e 9 acertos e 19,5\% entre 21 e 29 pontos. Apenas 5,2\% apresentou pontuações menores que 5 e $1,1 \%$ entre 30 e 33 pontos. Em relação à pontuação total do teste, que pode variar de 0 a 36 acertos, verificou-se que os participantes apresentaram média de 14,79 pontos, com $D P$ de 6,48, 15 pontos de mediana e 21 de moda. A menor pontuação encontrada foi de 1 acerto, e 33 foi a maior pontuação. As pontuações dos testes em relação à idade dos participantes podem ser observadas na Figura 2. 


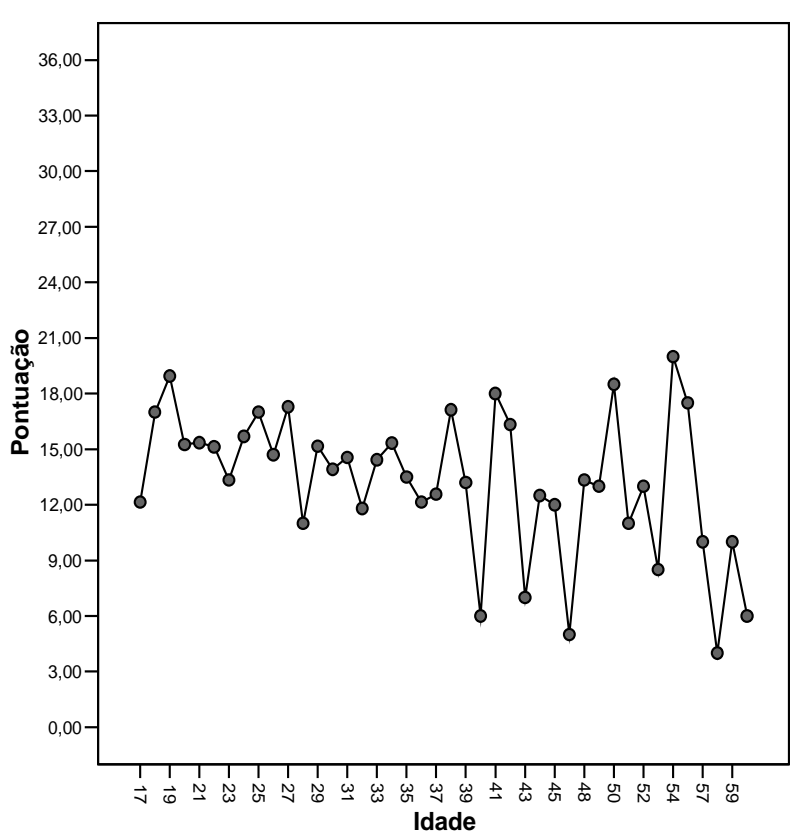

Figura 2 - Distribuição de pontos em relação à idade

No que tange aos estudos relacionados à variável idade, verificou-se uma leve diminuição das pontuações no teste com o avanço da idade, contudo observaram-se picos de pontuação nas idades mais avançadas. Realizou-se por meio da correlação de Pearson um estudo com a variável idade e a pontuação (acertos) no teste, entretanto foi encontrada uma fraca correlação negativa $(r=-0,13)$, que não demonstrou diferença estatística significante.

Em seguida, estudou-se a variação entre as médias nos testes em relação às idades dos respondentes, por meio da divisão da amostra por faixa etária, em quatro grupos de participantes. O grupo 1 correspondeu às idades entre 17 e 25 anos $(\mathrm{N}=178)$, com uma média de 15,63 (DP=6,54); o grupo 2 foi formado por pessoas entre 26 e 35 anos $(\mathrm{N}=126)$, com uma média de 14,18 (DP=6,11); o grupo 3 foi composto por idades entre 36 a 45 anos $(\mathrm{N}=40)$, cuja média foi de 13,85 ( $D P=6,97)$; por último o grupo 4, de 46 a 63 anos $(\mathrm{N}=25)$, correspondendo a uma média de 13,32 pontos $(D P=6,59)$. A Figura 3 apresenta a média de pontuação dos participantes no Raven Avançado por grupo de idade.

Verificou-se a ocorrência de médias mais elevadas nas idades entre 17 e 25 anos. Destacou-se que à medida que a idade avança as médias nas pontuações diminuem, uma vez que houve uma leve diminuição entre as médias da pontuação do teste em relação aos grupos de idades dos participantes. Mediante o objetivo de explorar diferenças relacionadas à variável idade, recorreu-se à análise de variância (ANOVA) para descobrir se existem diferenças entre as médias dos grupos, por meio do cálculo inicial da média geral dos grupos e da verificação do quanto a média de cada grupo difere da média geral (Dancey \& Reidy, 2006). Nos resultados obtidos pode-se observar que mesmo havendo diferenças entre as médias dos grupos de idade $(F=2,09$ e $p<0,100)$, tais diferenças não apresentam valores consideráveis estatisticamente significativos.

Buscou-se, ainda, verificar as diferenças nas pontuações do teste em relação ao gênero dos participantes. Os resultados revelaram que o sexo masculino apresentou uma média de 17 acertos e $D P$ igual a 6,38, sendo esta média superior a das mulheres, que obtiveram 13,92 acertos e DP igual a 6,31. Procurando verificar o quão relevante foi a diferença encontrada, realizou-se uma comparação de médias (Independent-Samples, $T$ Test) entre as pontuações encontradas no instrumento. Nos resultados obtidos destaca-se uma diferença de média entre homens e mulheres de 3,08 pontos $(t=4,21 \mathrm{e}$ $p<0,01)$.

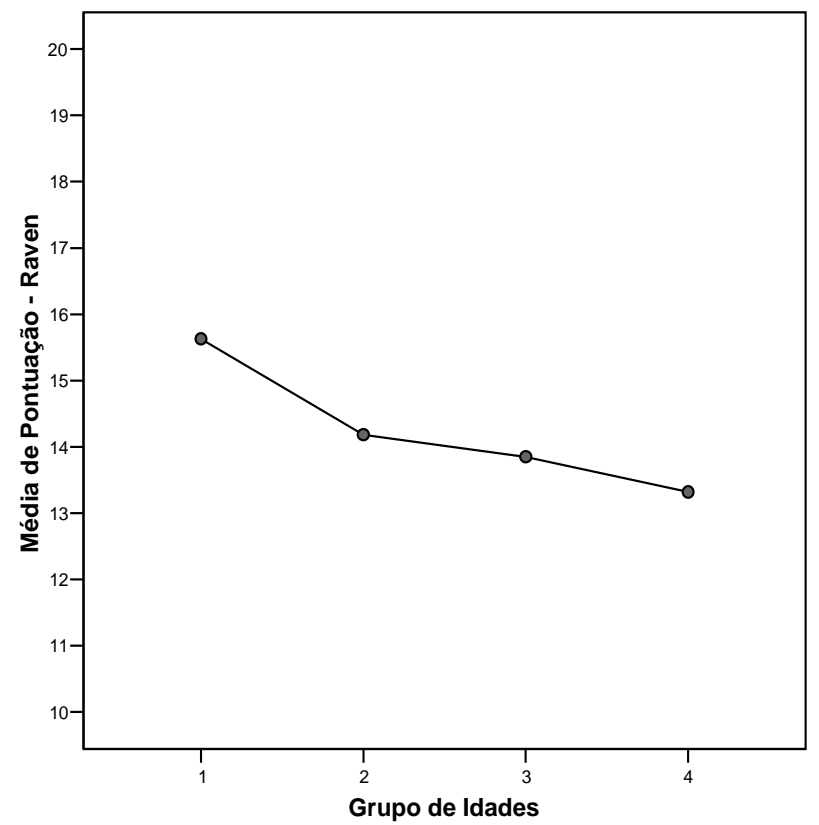

Figura 3. Média de pontuação dos participantes por grupo de idades

Vale mencionar a importância de análises relacionadas à idade, sexo, escolaridade, curso, entre outros, para o estudo de evidências de validade baseadas nas relações com outras variáveis, pois além de fornecer mais informações sobre as propriedades psicométricas, propiciam informações acerca da relação de determinadas variáveis e, em alguns casos, 
informam o quanto as possíveis diferenças podem ser atribuídas ao acaso. Nesse sentido, também foi realizada uma verificação da pontuação do teste em relação aos cursos que os participantes frequentavam. Os dados indicaram que o curso de Pedagogia obteve 11,35 ( $D P=5,41)$, o curso de Gestão em Recursos Humanos apresentou uma média de 11,50 pontos $(D P=5,80)$, o de Administração obteve uma média de $14,79(D P=6,31)$ e o de Psicologia, 16,60 $(D P=6,32)$. Com o objetivo de explorar análises de diferenças relacionadas à variável curso, recorreu-se à análise de variância (ANOVA). Nos resultados observou-se que ocorreram diferenças entre as médias dos grupos dos cursos $(F=13,8$ e $p<0,00)$.

Para identificar quais cursos se diferenciaram, realizou-se uma análise utilizando o teste ad-hoc de Tukey, adotando-se o nível de significância de $p=0,05$. Os subconjuntos formados estão apresentados na Tabela 1.

Tabela 1 - Agrupamento em razão das médias de pontos

\begin{tabular}{lccc}
\hline \multicolumn{1}{c}{ Cursos } & N & Grupo 1 & Grupo 2 \\
\hline Pedagogia & 37 & 11,35 & \\
Gestão RH & 54 & 11,50 & \\
Administraçã & 111 & & 14,79 \\
O & & & \\
Psicologia & 167 & & 16,60 \\
\hline Nota: $p<0,05$ & & &
\end{tabular}

Os resultados indicaram que os cursos de administração e psicologia obtiveram pontuações mais elevadas, evidenciando que os participantes desses grupos tiveram um menor número de erros, diferenciando-se significativamente dos demais. No que se refere à diferença entre as médias dos alunos de pedagogia e psicologia, observou-se uma diferença em torno de 5 pontos. Assim, verificou-se que os estudantes de psicologia obtiveram pontuações maiores e estatisticamente significativas.

\section{Discussão}

Este trabalho contribui para estudos com as Matrizes Progressivas Avançadas de Raven em universitários, e seus achados são coerentes com a literatura sobre o tema. Os resultados permitiram afirmar que, embora seja um instrumento que está em estudo de padronização e validação na população brasileira, o APM se mostrou uma medida confiável do respectivo construto no grupo analisado. Com relação à frequência de acertos no instrumento, observou-se que $56,1 \%$ permaneceram entre 10 e 20 acertos, o que possibilita analisar que a maioria dos participantes obteve um desempenho médio no instrumento, levando em consideração que quanto maior a pontuação melhor o desempenho e que a pontuação pode chegar até 36 pontos. Ressalta-se que nenhum participante alcançou pontuação maior que 33 acertos.

No que tange aos estudos relacionados à variável idade, verificou-se uma leve diminuição das pontuações no teste com o avanço da idade, contudo observaram-se picos de pontuação nas idades mais avançadas e a ocorrência de médias mais elevadas entre 17 e 25 anos. Destacou-se que, à medida que a idade avança, as médias nas pontuações diminuem, uma vez que houve uma leve diminuição entre as médias da pontuação do teste em relação aos grupos de idades dos participantes. Esses dados permitiram verificar uma tendência entre o avanço da idade e o declínio na pontuação nessa amostra, entretanto este dado não foi estatisticamente significativo. Esse dado também foi observado no estudo de Cunha (1974), mas questionado no estudo de Matarazzo (1976), que ressaltou a influência do tempo de escolarização o qual costuma ser diferente em indivíduos mais velhos, o que poderia influenciar a relação entre o desempenho no teste e a idade, pois muitos desses indivíduos mais velhos viveram em um contexto educacional diferente.

Com relação ao gênero dos participantes e às pontuações do teste, destacou-se uma diferença de média significativa de 3,08 pontos para o sexo masculino. Esses dados permitem inferir que a variável sexo influencia o desempenho dos indivíduos no APM. Dados como o do estudo de Weiss e colaboradores (2003) mostraram que os homens tendem a superar mulheres em tarefas espaciais visuais, enquanto nos testes verbais as mulheres apresentaram um nível mais elevado. Contrariando esses resultados, o estudo de Colom e Garciápz (2002) demonstrou não haver diferença entre gêneros no que tange aos aspectos de avaliação da inteligência.

No que se refere ao desempenho no teste por cursos, verificou-se que os estudantes de psicologia e de administração obtiveram pontuações maiores quando comparados com outros cursos e que essa diferença apresentou-se estatisticamente significativa. Vale salientar a possibilidade de que as médias mais baixas obtidas nos outros grupos de alunos possam estar relacionadas ao pouco comprometimento dos estudantes dos cursos de pedagogia e gestão em recursos humanos na execução do teste, entretanto, somente um reteste poderia verificar melhor essa hipótese.

Nesse sentido, é preciso considerar os argumentos de Candeias e Almeida (2000) no que se 
referem às diferenças individuais no desempenho intelectual, que também estão ligadas à relação da inteligência com o meio cultural e aos contextos de vida de cada pessoa. É inegável o reflexo de processos como motivação e criatividade no desempenho cognitivo dos indivíduos, mostrando que os níveis de habilidade e de desempenho realmente encontram-se diferenciados e se apresentam interligados às exigências decorrentes das mudanças culturais em torno da educação, do emprego, da qualidade de vida, entre outros, o que justifica um novo olhar em estudos sobre a inteligência. Ao se considerar a capacidade edutiva e reprodutiva do fator $\mathrm{g}$, pode-se ter, então, uma forte participação na capacidade reprodutiva exigida pelo APM dessas diferenças individuais, uma vez que nesta capacidade estão incluídos o domínio, a lembrança e a reprodução de materiais que constituem uma base cultural de conhecimentos explícitos.

\section{Considerações finais}

Para futuros estudos algumas limitações metodológicas podem ser superadas, como, por exemplo, a comparação de estudantes dos mesmos cursos em diversas universidades de várias regiões do País, e assim aumentar a amostra de estudantes entre os cursos para que a pesquisa se torne mais representativa. Diante de tais considerações, novas pesquisas podem ser realizadas, proporcionando um maior subsídio na investigação desses dados para a população brasileira.

Ao término dessas análises espera-se contribuir com o conhecimento sobre o tema e, mais especificamente, com relação aos estudos sobre as propriedades psicométricas do teste Matrizes Progressivas Avançadas de Raven - APM, uma vez que este demonstrou ser um instrumento preciso para avaliar aspectos da inteligência. Entretanto, chama-se a atenção para o fato de que a avaliação do desempenho de indivíduos com grau de inteligência superior ao da população geral, mediante normas atuais, poderia beneficiar o trabalho de profissionais que atuam em seleção de pessoal, por exemplo, uma vez que possibilitaria o acesso a um instrumento que permitiria uma classificação da inteligência mais adequada para essa população.

\section{Referências}

Alves, I. C. B. (1998). Variáveis significativas na avaliação da inteligência. Psicologia Escolar $e$ Educacional, 2(2), 1-7.
Alves, I. C. B. (2006). Novos estudos psicométricos do Teste D.70. Avaliação Psicológica, 5(2), 251-253.

Alves, I. C. B. (2007). Teste D.70: manual revisado e ampliado. São Paulo: CETEPP.

Alves, I. C. B., Colosio, R. \& Ruivo, R. (1992). O Teste R1: um estudo das variáveis idade, sexo e escolaridade. Anais da $44^{\circ}$ Reunião Anual da Sociedade Brasileira para o Progresso da ciência, São Paulo, 44, 904.

Anastasi, A. (1977). Testes psicológicos. São Paulo: EPU.

Angelini, A. L., Alves, I. C. B., Custódio, E. M., Duarte, W. F. \& Duarte, J. L. M. (1999.) Matrizes Progressivas Coloridas de Raven: escala especial. Manual. São Paulo: CETEPP.

Candeias, A. M. \& Almeida, L. S. (2000). Contributos da inteligência social ao estudo da inteligência e da sobredotação. Revista Sobredotação, 1(2), 129-146.

Colom, R. \& Garciápz, O. (2002). Sex differences in fluid intelligence among high school graduates. Personality and Individual differences, 32, 445-451.

Conselho Federal de Psicologia - CFP (2007). Sistema de Avaliação de testes psicológicos. Obtido em 03 de maio de 2007 do World Wide Web: http:/ /www.pol.org.br/satepsi

Cronbach, L. J. (1996). Fundamentos da testagem psicológica. $5^{\text {a }}$ edição. Porto Alegre: Artes Médicas.

Cunha, S. E. (1974). A psicometria da inteligência e a dimensão idade. Arquivos Brasileiros de Psicologia Aplicada, 26(3), 100-110.

Dancey, C. P. \& Reidy, J. (2006). Estatística sem matemática para psicologia. Porto Alegre: Artmed.

Matarazzo, J. (1976). Wechsler: medida e avaliação da inteligência do adulto. São Paulo: Manole.

Muñiz, J. (Org.). (1996). Psicometria. Madrid: Universitas.

Noronha, A. P. P. \& Alchieri, J. C. (2002). Reflexões sobre os instrumentos de avaliação psicológica. Em R. Primi (Org.). Temas em avaliação psicológica (p. 19-23). Campinas: Impressão Digital do Brasil. 
Noronha, A. P., Sartori, F. A., Freitas, F. A. \& Ottati, F. (2001). Informações contidas nos manuais de testes de inteligência publicados no Brasil. Psicologia em Estudo, 6(2), 101106.

Paín, S. (1992). Psicometria genética. São Paulo: Casa do Psicólogo.

Pasquali, L. (2001). Técnicas de exame psicológico (TEP) - manual: fundamentos das técnicas psicológicas (v. 1). São Paulo: Casa do Psicólogo, Conselho Federal de Psicologia.

Pasquali, L. (2002). Matrizes Progressivas do Raven Infantil: um estudo de validação para o Brasil. Avaliação Psicológica, 2, 95-110.

Primi, R. \& Almeida, L. (2000). Estudo de validação da Bateria de Provas de Raciocínio (BPR - 5). Psicologia: Teoria e Pesquisa, 16(2), 165-173.

Primi, R., Flores-Mendoza, C. E. \& Castilho, A. V. (1998). Estudo exploratório das propriedades psicométricas do Teste de
Raven Escala Avançada Informatizado. Boletim de Psicologia, 48, 67-75.

Raven, J., Raven J. C. \& Court, J. H. (1998). Manual for Raven's Progressive Matrices and Vocabulary scales: Section 4 advanced progressive matrices sets I \& II. Oxford: Oxford Psychologysts Press.

Wechsler \& R. S. L. Guzzo (Orgs.). Avaliação psicológica: perspectiva internacional (p. 133141). São Paulo: Casa do Psicólogo.

Wechsler, S. M. (1999). Guia de procedimentos éticos para a avaliação psicológica. Em S. M.

Weiss, E. M., Kemmler, G., Deisenhammer, E. A., Fleischhacker, W. W. \& Delazer, M. (2003). Sex differences in cognitive functions. Personality and Individual Differences, 35 863-875.

Recebido em julho de 2008 Reformulado em fevereiro de 2009 Aprovado em março de 2009

Sobre os autores:

Milena Oliveira Rosseti é psicóloga, mestranda em Psicologia Clínica no Instituto de Psicologia da Universidade de São Paulo e pesquisadora do Departamento de Pesquisa e Produção de Testes da Casa do Psicólogo.

Ivan Sant'Ana Rabelo é psicólogo, mestre em Avaliação Psicológica pela Universidade São Francisco/Itatiba e Pesquisador do Departamento de Pesquisas e Produção de Testes da Casa do Psicólogo.

Irene F. Almeida de Sá Leme é psicóloga com especialização em Recursos Humanos pela Fundação Getúlio Vargas- FGV/SP, docente das disciplinas Psicologia e Ética e pesquisadora do Departamento de Pesquisa e Produção de Testes da Casa do Psicólogo.

Sílvia Verônica Pacanaro é psicóloga, mestre em Avaliação Psicológica pela Universidade São Francisco/Itatiba. Especialista em Psicopedagogia pela PUC-CAMPINAS e Pesquisadora do Departamento de Pesquisas e Produção de Testes da Casa do Psicólogo.

Ingo Bernd Guntert é psicólogo e diretor do Departamento de Pesquisa e Produção de Testes da Casa do Psicólogo. 